\title{
A tabu search for multi-objective single row facility layout problem
}

N.Lenin ${ }^{1}$, M.Siva Kumar ${ }^{2}$, D.Ravindran ${ }^{1}$ and M.N.Islam ${ }^{3}$

${ }^{1}$ Department of Mechanical Engineering, National Engineering College, K.R.Nagar, Kovilpatti, Tamilnadu - 628 503, India.

${ }^{2}$ Sree Sowdambika College of Engineering, Chettikurichi, Aruppukottai, Tamilnadu - 626 134, India.

${ }^{3}$ Department of Mechanical Engineering, Curtin University, GPO Box U 1987, Perth WA 6845, Australia.

Corresponding author:

Dr. M. Siva Kumar, Principal,

Sree Sowdambika College of Engineering,

Chettikurichi, Aruppukottai,

Tamilnadu - 626 134, India.

Email_ : lawan_sisa@yahoo.com

Mobile : : +91-9659599970

Telephone : : +91 -04566-229805 


\title{
A tabu search for multi-objective SRFLP
}

\begin{abstract}
This paper addresses the problem of multi objective facility layout planning. The aim is to solve the single row facility layout problems (SRFLP) and find the linear machine sequence which minimizes the following: the total investment cost of machines; the total material handling cost; the total number of machines in the final sequence; and the total flow distance of the products in units. The tabu search algorithm (TSA) which has now become a very useful tool in solving a variety of combinatorial optimization problems is made use of here. TSA is developed to determine the product sequence based on which a common linear machine sequence is found out for multi-products with different machine sequences. We assume that, limited number of duplicate machine types available for job. The results are compared with other approaches and it shows the effectiveness of the TSA approach as a practical decision support tool to solve problems in SRFLP.
\end{abstract}

Keywords: SRFLP; linear sequencing of machines; tabu search algorithm; investment cost of machines; material handling cost; flow distance.

\section{Introduction:}

The most decision makers face a common problem in any industrial setup is the finalization of its layout, i.e. deciding the location of the machines with respect to all products' sequences (Suresh and Sahu 1993). The multi product flow lines concept is a very big challenge to the researchers. It facilitates different products to be produced simultaneously in a single setup of a flowline, which in turn increase the utilization of the manufacturing process (Bhaba R. Sarker 2003). The machine layout or flowline design involves determination of the relative positions of the machines in a layout.

A linear machine sequence is the most popular among production systems due to its simplicity and efficient flow structure (Heragu and Kusiak 1990, Kouvelis and Chiang 1992), as well as for its ability to arrange machines in a variety of flow configurations such as ${ }^{*}$ straight line, U-shape line, serpentine line or loop for a conveyor or an automated guided vehicle (AGV) system (Aneke and Carrie 1986). It has the benefits of shorter flow distance, easier control of the production process and easier material handling. This type of layout configuration is the most commonly used form in cellular manufacturing systems (Nicholas 2001, Zhou and Irani 2003) and flexible manufacturing systems (Houshyar and McGinnnis 1990, Kouvelis and Kim 1992, Lee et al. 2001). Hence, in this work, a linear machine sequencing method has been selected.

This paper is divided into the following sections. Section 2 reviews the literature and section 3 presents the problem background and mathematical model of finding the

\footnotetext{
*Corresponding Author. Email: lawan_sisa@yahoo.com
} 
objectives. The proposed heuristic and TSA are discussed in section 4. In section 5 the Numerical illustration for the proposed method is provided. Computational results are discussed in section 6. Conclusion and future research is presented in section 7.

\section{Literature review:}

The theoretical attractiveness and practical applications of the problem have created a rich and growing literature for the SRFLP, which has proven to be a NP-complete problem (Suresh and Sahu, 1993). Since SRFLP is NP-complete, the application of exact methods to large instances of the problem is too time consuming; therefore, heuristic methods have been developed to obtain a near optimal solution of the problem.

Many researchers have developed the heuristic methods in the formulation of linear sequencing of machines for SRFLP.

Heragu and Kusiak (1990) presented two efficient models, namely, a linear continuous and linear mixed integer, for facility layout problems. The models do not necessitate prior knowledge of site locations. The authors showed that the continuous models are more useful for solving facility layout problems than are other models presented in literature.

Houshyar and McGinnis (1990) introduced a heuristic for assigning facilities to locations to minimize work in process travel distance in a straight track. Authors established the performance of their heuristic better than the modified and classical lower bound methods for the test problems.

Heragu and Alfa (1992) experimentally analyzed simulated annealing-based algorithms, namely, a modified penalty algorithm, the simulated annealing algorithm, and a hybrid simulated annealing algorithm for single-row layout problems in facilities of unequal areas and for multi-row layout problems in facilities of equal areas. The authors concluded that the hybrid algorithm produces better quality solutions than do the first two algorithms, although the former involves slightly longer computational time.

Kouvelis and Chiang (1992) implemented a simulated annealing procedure to determine a flow line (or single-row layout) under the assumptions that the number of machines is fixed and backtrack movements are allowed. The authors aimed to determine a machine sequence with minimum total backtrack distance. Booncharoen Sirinaovakul (1996) gave an idea of how the facility layout algorithm can be constructed and how the quality of the facility layout algorithm can be improved.

Wen-Chyuan Chiang (1997) implemented a tabu search heuristic, a probabilistic tabu search heuristic, a simulated annealing heuristic and a hybrid tabu search heuristic to minimize the material handling cost in facility layout problems with the quadratic assignment problem formulation.

Braglia (1997) regarded the linear machine sequencing problem as a nonpolynomial hard combinatorial problem. The number of possible sequences grows exponentially because the use of duplicate machines is allowed. Moreover, the set of all feasible sequences is not merely a set of simple permutations of a fixed number of machines given that the sequences must satisfy the different operation sequences of all products. The author determined a linear machine sequence with minimum expected movement of the machine handling device located between machines in a machine cell. The expected movement is determined by the frequency of part displacements between machines. 
Wang et al., (1998) formulated a model for minimizing the total material handling distance on a shop floor in both inter- and intra-cell facility layouts for cellular manufacturing systems. The authors used an improved simulated annealing algorithm to solve this problem.

Ho and Moodie (1998) investigated a machine layout problem with a linear single-row flow line for an automated manufacturing system. The authors also investigated the effect of flow line characteristics on machine layouts. They provided vital information on selecting appropriate flow line analysis methods and determining appropriate evaluation criteria for different layout problems.

Chen et al (2001) addressed the problem of determining a common linear machine sequence for multi-products that have different operation sequences and facilities with a limited number of duplicate machine types. The authors intended to minimize the total flow distance travelled by products on this linear flow line by using a modified simulated annealing algorithm.

Housni Djellab and Michel Gourgand (2001) proposed a new heuristic to minimize the total time required by material handling systems to transport the part types between machines in a single-row facility layout problem arising in Flexible Manufacturing Systems.

Diponegoro and Sarker (2003) presented a two-stage solution methodology that simplifies computation and generates better solutions for reducing travel distances in production processes that involve sets of identical machines. This problem is often formulated as a tertiary assignment problem because of its combinatorial nature. According to Hicks (2004), layouts produced by a genetic algorithm-based optimization method significantly minimize material movement for a given work schedule in both greenfield and brownfield scenarios.

Chrysostomos and Vlachos (2005) used the linear programming model for minimal backward flow to determine the optimal linear machine sequence in a manufacturing cell. They applied a modified ACS algorithm to the conditions and parameters of the linear machine layout problem. To determine the optimal linear placement of facilities with varying dimensions on a straight line, Anjosa et al. (2005) introduced a semi-definite programming approach for the one-dimensional spaceallocation problem, also known as the single-row facility layout problem.

Pillai et al (2005) identified a linear sequence that minimizes the total distance travelled by multiple items with different operation sequences. The authors regarded each type of machine available as limited, and adopted a simulated annealing algorithm in determining the best solution. Solimanpur (2005) et al. formulated the single-row machine layout problem as a non-linear 0-1 programming model, in which the distance between the machines is sequence dependent. They developed an ant colony algorithm to solve this problem.

Singh and Sharma (2006) discussed the current and future trends of research on facility layout problems. The authors observed a trend toward multi-objective approaches by developing facility layout software using meta-heuristics, such as simulated annealing, genetic algorithm, and concurrent engineering for facility layouts. Andre and Amaral (2008) proposed a mixed 0-1 linear program for the one-dimensional facility layout problem to minimize the weighted sum of the distances.

Lou Y. Liang and Wen C. Chao (2008) developed a tabu search algorithm to improve the layout in the facility layout problem. The results i.e., the costs on the facility layout arrangement and preferences by the tabu search are better when 
compared with genetic algorithm and artificial neural network. Teo and Ponnambalam (2008) proposed a hybrid ACO/PSO heuristic to solve single-row layout problems. For apparel manufacturing, Lin (2009) proposed a hierarchical order-based genetic algorithm to minimize the moving distance among cutting pieces in a U-shaped singlerow machine layout.

Ramazan Şahin and Orhan Türkbey (2009) considered the facility layout problem which combines the objective of minimization of the total material handling cost and the maximization of total closeness rating scores. A simulated annealing (SA) algorithm was proposed to find the non-dominated solution (Pareto optimal) set approximately for the multi-objective facility layout problem. The Pareto optimal sets generated by the proposed algorithm were compared with the solutions of the previous algorithms for multi-objective facility layout problem. The results showed that the approximate Pareto optimal sets found include almost all the previously obtained results and many more approximate Pareto optimal solutions.

Hamed Samarghandi and Kourosh Eshghi (2010) proposed a new algorithm based on tabu search for the special case of SRFLP to find an optimal linear placement of rectangular facilities with varying dimensions on a straight line. Computational results of the proposed algorithm on benchmark problems show the greater efficiency of the algorithm compared to the other heuristics for solving the SRFLP. Computational results of the proposed algorithm on benchmark problems showed the greater efficiency of the algorithm compared to the other heuristics for solving the SRFLP.

Satheesh Kumar et al. (2010) employed an artificial immune system algorithm to minimize material handling costs both in single-row and loop layout problems in FMSs. Siva Kumar et al. (2011) developed a simple heuristic to determine the optimal linear sequence that minimizes the flow distance travelled by products.

Even with the significant attempt directed toward solving SRFLP, most of these studies focused on the optimization of a single parameter only-flow distance. In practice, however, the total number of machines in a layout and the total investment cost of machines and material handling cost are equally important factors. To the best of our knowledge, there is no published work in SRFLP to minimize flow distance, number of machines, machines cost and material handling cost by using TSA. In the spirit of the above considerations, and to add to the evaluation of TSA approaches to this complex problem, this paper proposes TSA to provide optimal solution for SRFLP.

\section{Mathematical formulation:}

\subsection{Problem background:}

The location and number of machines in a linear machine sequence of a single row facility layout design are the keys to determine the flow distance of multi-products, total investment cost of machines and total material handling cost. In facilities with duplicate machines and multiple products, the single-row layout design is considered a nonpolynomial hard problem [Braglia (1997)].

The assumptions considered in the proposed method are:

(1) The number of products, flow distance of products, machine type sequences, and individual cost of the machine types will be known along with the availability of duplicate machine types. 
(2) The products are entered exactly of their first machine given in the final linear machine sequences.

(3) The products' flow distances are considered up to the end machine type of the respective products without affecting the precedence.

(4) No machines will be repeated adjacently in the final linear machine sequence.

(5) Backtracking is not permitted.

\section{2 Objective functions and models:}

\subsubsection{Total flow distance in units:}

The total flow distance of a product in units $(t d)$ is determined using equation (1). The constraints are presented in equations (2)-(6):

$$
t d=\sum_{i=1}^{n p} \sum_{j=1}^{n m_{i}} d_{i}\left(L_{i j+1}-L_{i j}\right)
$$

where

td $\quad-$ total flow distance

$d_{i} \quad-\quad \mathrm{i}^{\text {th }}$ product flow distance

$L_{i j+1} \quad-i^{\text {th }}$ product's $j+1^{\text {th }}$ machine location in the final machine sequence

$L_{i j} \quad-\quad \mathrm{i}^{\text {th }}$ product's $\mathrm{j}^{\text {th }}$ machine location in the final machine sequence

$n p \quad$ - number of products

$n m_{i} \quad$ - number of machines in $i^{\text {th }}$ product's machine sequence.

$$
\begin{aligned}
& L_{i j+1}>L_{i j} \\
& L_{i j}>L_{i 1} \\
& n m_{k} \leq n d m_{k}
\end{aligned}
$$

where

$n m_{k} \quad$ - number of $\mathrm{k}^{\text {th }}$ machine available in the final linear machine sequence $n d m_{k}-$ number of duplicate $\mathrm{k}^{\text {th }}$ machine type available for usage.

Equation (2) shows that the location of the $j+1^{\text {th }}$ machine should always be larger than the location of the $j^{\text {th }}$ machine in the linear machine sequence. Equation (3) indicates that the location of the $j+1^{\text {th }}$ machine in the individual product machine sequence should always be larger than the location of the first machine in the linear machine sequence. According to equation (4), the number of $k^{\text {th }}$ machines types available in the final linear machine sequence should be less than or equal to the number of duplicate $k^{\text {th }}$ machine types available for use.

\subsubsection{Total number of machines in the final linear sequence:}

The minimum number of machines in the final linear sequence (nms) of the single-row layout design reduces both flow distance and initial investment. This is expressed using 


$$
n m s=\operatorname{count}(b[\ldots . . .])
$$

where $b[\ldots . .$.$] represents the final linear machine sequence.$

$$
t m=\sum_{k=1}^{n m t} n d m_{k}
$$

where

tm - total number of machines available for use

nmt - number of machine types

$k \quad-$ index to represent the machine type $k=1,2,3, \ldots . n m t$.

where

$$
n m s \leq t m
$$

nms - total number of machines available in the final linear sequence.

The total number of machines is equal to the sum of the duplicates of individual machine types; this total is given in equation (6). Equation (7) shows that the total number of machines in the final linear sequence must be less than or equal to the total number of machines available for use, including the duplicate machines.

\subsubsection{Total investment cost of machines:}

Companies prefer to reduce not only their operation/manufacturing costs but also their initial investment. In the single-row layout design, the investment cost of machines is expressed by

$$
t c=\sum_{k=1}^{n m t} c_{k} n m_{k}
$$

Where,

tc - total investment cost of machine in the final linear sequence

$c_{k} \quad-$ cost of the $\mathrm{k}^{\text {th }}$ machine type.

\subsubsection{Total material handling cost:}

Tompkins et al (1996) estimated that between $20 \%$ and 50\% of total operating cost with in manufacturing can be attributed to material handling. So, the minimization of material handling cost is also an equal important factor. The estimation of total material handling cost is detailed in equation (9).

$$
t m h c=\sum_{i=1}^{n p} \sum_{j=1}^{n m_{i}} m h c_{i j-i j+1}
$$

Where,

tmhe $\quad-$ total material handling cost of all products in the final linear 


$$
\begin{array}{ll} 
& \text { sequence } \\
m h c_{i j-i j+1} & - \text { material handling cost between } \mathrm{j}^{\text {th }} \text { machine to } \mathrm{j}+1^{\text {th }} \text { machine } \\
& \text { for the } \mathrm{i}^{\text {th }} \text { product in the final linear sequence }
\end{array}
$$

\subsubsection{Average fitness factor:}

The total flow distance in units, total number of machines in the final linear sequence, total investment cost of machines and total material handling cost are at different ranges or levels. Summing up the above-mentioned values at different levels will not produce the best result. We therefore apply the average fitness factor method [Sivakumar $\mathrm{K}$ et al. (2011)] to derive the objective values within the range of 0 to 1 (normalaization). The normalized values of total flow distance, total number of machines, investment cost of machines, and material handling cost are determined using equations (10)-(13).

$$
\begin{aligned}
n t d_{l} & =\frac{t d_{\text {max }}-t d_{l}}{t d_{\text {max }}-t d_{\min }} \\
n n m s_{l} & =\frac{n m s_{\text {max }}-n m s_{l}}{n m s_{\text {max }}-n m s_{\min }} \\
n t c_{l} & =\frac{t c_{\text {max }}-t c_{l}}{t c_{\max }-t c_{\min }} \\
n t m h c_{l} & =\frac{t m h c_{\max }-t m h c_{l}}{t m h c_{\max }-t m h c_{\min }}
\end{aligned}
$$

\begin{tabular}{|c|c|c|}
\hline$n t d_{l}$ & - & $\begin{array}{l}\text { normalized value of total flow distance of multi } \\
\text { products for the } l^{\text {th }} \text { sequence of products. }\end{array}$ \\
\hline$n n m s_{l}$ & - & $\begin{array}{l}\text { normalized value of total number of machines in the } \\
\text { final linear machine sequence of } l^{\text {th }} \text { sequence of products. }\end{array}$ \\
\hline$n t c_{l}$ & - & $\begin{array}{l}\text { normalized value of total investment cost of machine } \\
\text { for the } l^{\text {th }} \text { sequence of products. }\end{array}$ \\
\hline$n t m h c_{l}$ & - & $\begin{array}{l}\text { normalized value of total material handling cost of } \\
\text { multi products for the } l^{\text {th }} \text { sequence of products. }\end{array}$ \\
\hline$t d_{\min } \& t d_{\max }$ & - & $\begin{array}{l}\text { minimum \& maximum value of total flow distance for } \\
1,2,3, \ldots l \text { number of sequences of products. }\end{array}$ \\
\hline $\begin{array}{l}n m s_{\min } \& \\
n m s_{\max }\end{array}$ & - & $\begin{array}{l}\text { minimum \& maximum number of machines in the final } \\
\text { linear sequence for } 1,2,3, \ldots l \text { number of sequences of } \\
\text { products. }\end{array}$ \\
\hline$t c_{\min } \& t c_{\max }$ & - & $\begin{array}{l}\text { minimum \& maximum value of total investment cost of } \\
\text { machines for } 1,2,3, \ldots l \text { number of sequences of products. }\end{array}$ \\
\hline \multicolumn{3}{|l|}{$t m h c_{\min } \&$} \\
\hline$t m h c_{\max }$ & - & $\begin{array}{l}\text { minimum \& maximum value of total material handling } \\
\text { cost for } 1,2,3, \ldots l \text { number of sequences of products. }\end{array}$ \\
\hline$d_{l}$ & - & total flow distance of multi products for the $l^{\text {th }}$ \\
\hline
\end{tabular}

Where, 


$\begin{array}{lll}n m s_{l} & - & \begin{array}{l}\text { sequence of products. } \\ \text { total number of machines in final sequence of } l^{\text {th }} \\ \text { sequence of products. }\end{array} \\ t c_{l} & - & \begin{array}{l}\text { total investment cost of machines for the } l^{\text {th }} \text { sequence of } \\ \text { products. } \\ t m h c_{l}\end{array} \quad-\quad \begin{array}{l}\text { total material handling cost of multi products for the } l^{\text {th }} \\ \text { sequence of products. }\end{array}\end{array}$

The average fitness factor value is determined by equation (14). In the minimization problem, the maximum value of average fitness factor is considered.

$$
n d m c_{l}=\frac{n t d_{l}+n n m s_{l}+n t c_{l}+n t m h c_{l}}{4}
$$

Where,

$n d m c_{l} \quad-\quad$ average fitness factor

The corresponding linear machine sequence of the above said minimum average fitness factor value is the optimum one among the $l$ number of sequences of products.

\section{Proposed methodology:}

The problem has been solved using a simple heuristic and tabu search. In which, tabu search helps to determine the product's sequence and the final linear machine sequence for the product's sequence is obtained using a simple heuristic.

\subsection{Simple heuristic to evaluate linear sequence of machines:}

A simple heuristic has been developed in this work to evaluate linear sequence of machines for the given product's sequence. This heuristic reduces the computation time and improve the consistency of the solutions. The detailed algorithm is given below.

Step 1: $\quad$ Read number of machine types $(\mathrm{nm})$, number of duplicate machine in each type (mtn[]), number of products $(n p)$, number of machine type in each product (nmp[]), machine type sequence for each product (pseq[][]), product sequence ( $p s[])$, flow distance of each product ( $p d[])$, cost of each machine type $(m c[])$ and material handling cost between machine to machine $(m h c[])$.

Step 2: Assign the machine type for the first product's machine sequence and store in $b[]$. Update the availability of the machine type in $m t n[]$.

Step 3: $\quad$ For each of the remaining product in pno[] do the steps 4 to 11.

Step 4: $\quad$ For each machine type (mno) in the product sequence pseq[] [] do step 5.

Step 5: If the machine mno is unassigned then add the machine in front of the existing machine sequence $b[]$ and update its availability. Go to step 4.

Step 6: If the machine type is assigned, check the machine type mno in the existing sequence $b[]$

Step 7: If available, check the availability of the remaining machine type of pseq[][] in $b[]$. 
Step 8: $\quad$ If all the machine types are available then go to step 3.

Step 9: If not, if the machine type mno is unassigned then insert the mno in the appropriate location (the location that doesn't affect the existing product machine sequence in $b[]$ ) after the location of the previous machine type exist in the existing machine sequence $b[]$ and update the availability. Go to step 4.

Step 10: If the machine type mno is unassigned but the insertion in $b[]$ affects the existing product sequence, then add the mno at the end of $b[]$ and update the availability. Go to step 4.

Step 11: If the machine type mno is assigned then the existing sequence is not feasible and stop the program.

Step 12: Display the linear sequence of machine type $b[]$.

\subsection{Tabu search algorithm:}

Tabu Search (TS) is a meta-heuristic approach that has been widely used in solving different combinatorial optimization problems [Glover and Laguna (1997)]. TSA is developed to identify a linear machine sequence among the alternatives which minimizes the following: the total investment cost of machines; the total material handling cost; the total number of machines in the final sequence; and the total flow distance of the products in units.

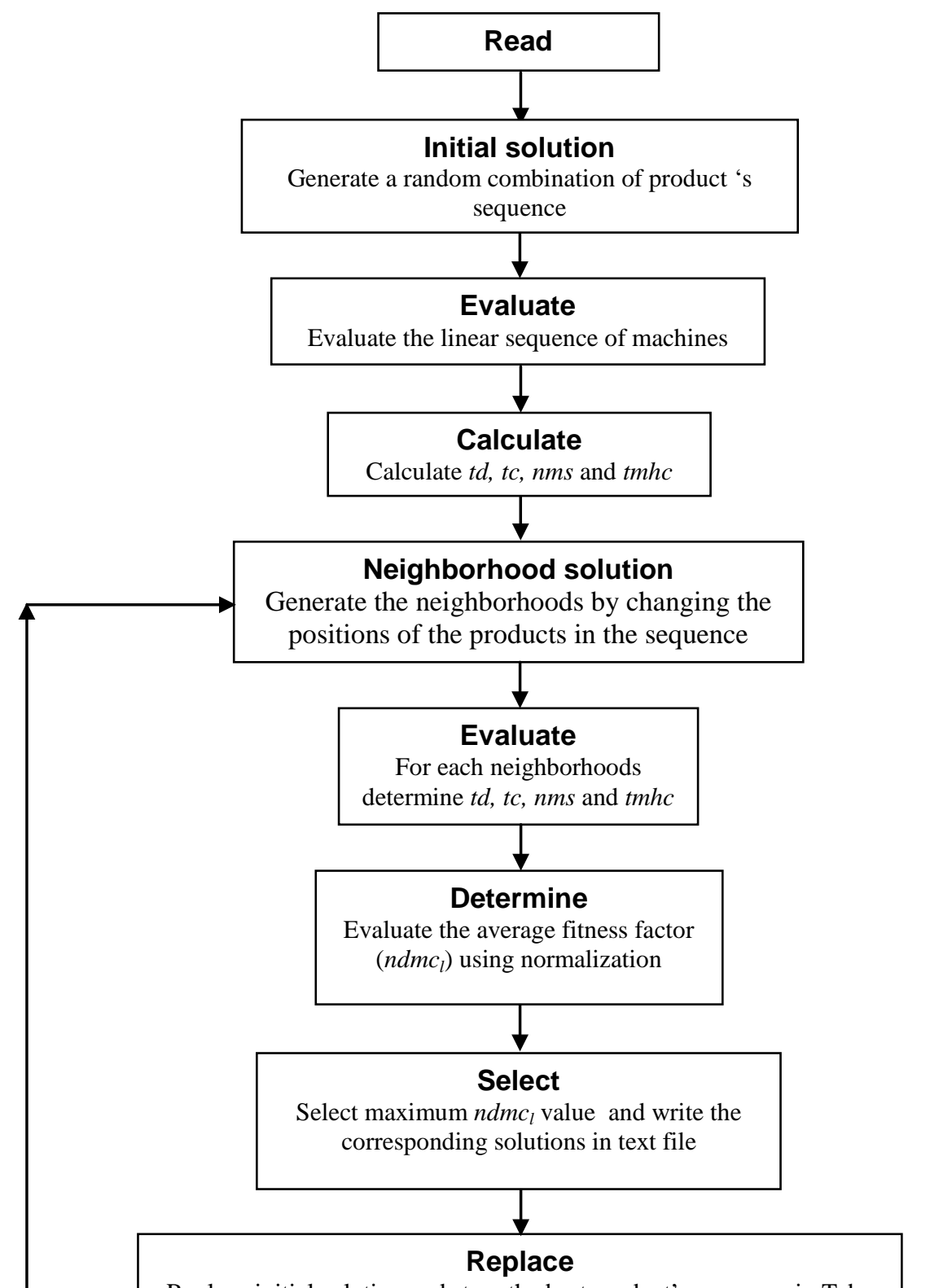


Figure 1. Scheme of tabu search algorithm.

In tabu search, a tabu list is a set of solutions determined by historical information from the last ' $t$ ' iterations of the algorithm, where ' $t$ ' is fixed or is a variable that depends on the state of the search, or a particular problem. At each iteration, given the current solution $\mathrm{x}$ and its corresponding neighborhood $\mathrm{N}(\mathrm{x})$, the procedure moves to the solution in the neighborhood $\mathrm{N}(\mathrm{x})$ that most improves the objective function. However, moves that lead to solutions on the tabu list are forbidden. If there are no improving moves, TS chooses the move which least changes the objective function value. The tabu list avoids returning to the local optimum from which the procedure has recently escaped. A basic element of tabu search is the aspiration criterion, which determines when a move is admissible despite being on the tabu list. One termination criterion for the tabu procedure is a limit in the number of consecutive moves for which no improvement occurs. figure 1.

The detailed step-by-step procedure of TSA for the proposed method is shown in

\section{Illustrative numerical example:}

The following example problem has been considered to illustrate the effectiveness of the proposed method. Table 1 shows the number of machine types (M.No.), its availability and investment cost.

Table 1. Details of machine, its availability and cost.

\begin{tabular}{cccccccccc}
\hline M.No. & 1 & 2 & 3 & 4 & 5 & 6 & 7 & 8 & 9 \\
\hline $\begin{array}{c}\text { Availability } \\
\text { of duplicate machines }\end{array}$ & 3 & 2 & 3 & 3 & 2 & 3 & 2 & 3 & 3 \\
$\begin{array}{c}\text { Machine cost } \\
\text { (Rs.) }\end{array}$ & 12554 & 32457 & 17685 & 19658 & 30254 & 22887 & 27889 & 19651 & 18357 \\
\hline
\end{tabular}


The product number (P.No.), individual product's machine type sequences and the demand of the product in units (flow distance) are represented in Table 2.

Table 2. Details of machine sequence and demand of individual product.

\begin{tabular}{clc}
\hline P.No. & \multicolumn{1}{c}{ Machine sequence } & Demand in units \\
\hline 1 & $9-8-1-2$ & 12 \\
2 & $4-5-2-3$ & 17 \\
3 & $1-7-6-2-4$ & 13 \\
4 & $3-6-5-1$ & 14 \\
5 & $4-3-2-7-6$ & 19 \\
6 & $6-7-8-5$ & 11 \\
7 & $1-2-3-4$ & 10 \\
\hline
\end{tabular}

The details of material handling cost between machine to machine is given in Table 3.

Table 3. Details of material handling cost between machine to machine.

\begin{tabular}{|c|c|c|c|c|c|c|c|c|c|c|}
\hline \multirow{2}{*}{\multicolumn{2}{|c|}{$\begin{array}{c}\text { Material } \\
\text { handling cost } \\
\text { in Rs. }\end{array}$}} & \multicolumn{9}{|c|}{ Machines } \\
\hline & & 1 & 2 & 3 & 4 & 5 & 6 & 7 & 8 & 9 \\
\hline \multirow{9}{*}{ 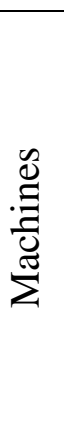 } & 1 & 0 & 41 & 34 & 49 & 54 & 45 & 58 & 49 & 53 \\
\hline & 2 & 58 & 0 & 46 & 32 & 35 & 32 & 62 & 52 & 56 \\
\hline & 3 & 58 & 52 & 0 & 65 & 54 & 42 & 60 & 37 & 46 \\
\hline & 4 & 31 & 32 & 37 & 0 & 31 & 53 & 58 & 33 & 58 \\
\hline & 5 & 69 & 55 & 38 & 48 & 0 & 44 & 45 & 53 & 35 \\
\hline & 6 & 55 & 51 & 49 & 57 & 64 & 0 & 62 & 65 & 63 \\
\hline & 7 & 38 & 63 & 55 & 61 & 36 & 33 & 0 & 47 & 55 \\
\hline & 8 & 68 & 55 & 67 & 33 & 41 & 32 & 32 & 0 & 50 \\
\hline & 9 & 33 & 53 & 65 & 47 & 52 & 41 & 61 & 50 & 0 \\
\hline
\end{tabular}

Initial solution 1-2-5-3-6-4-7 i.e. a random combination of product's sequence is generated randomly. The detailed step-by-step procedure to compute the machine sequence for the above mentioned product sequence is presented in the following section.

\subsection{Stepwise procedure to evaluate the linear machine sequence:}

For the combination of product sequence 1-2-5-3-6-4-7, the final linear machine sequence is evaluated by the following procedure. 
Step I: Select first product machine type sequence 9-8-1-2. Assign machine type 9,8,1 and 2. Store in b[]. Update the availability of machine types in table 1 and the same is given below in table 4 .

Table 4. Updated unassigned machine types.

\begin{tabular}{cccccccccc}
\hline Machine types & $\mathbf{1}$ & $\mathbf{2}$ & 3 & 4 & 5 & 6 & 7 & $\mathbf{8}$ & $\mathbf{9}$ \\
\hline $\begin{array}{c}\text { No of machines } \\
\text { available }\end{array}$ & $\mathbf{2}$ & $\mathbf{1}$ & 3 & 3 & 2 & 3 & 2 & $\mathbf{2}$ & $\mathbf{2}$ \\
\hline
\end{tabular}

The linear machine type sequence $(b[])$ after first product is $\mathbf{9 - 8 - 1 - 2}$.

Step II: Next $\left(2^{\text {nd }}\right)$ product machine type sequence is 4-5-2-3. Machine type number (mno) 4 is unassigned and unavailable (as per Step 5) in the existing sequence $b[]$, hence, add the machine types in front of $b[]$ and update the availability of machine type. Similarly, the other machine types 5,2 and 3 are unassigned and hence, add in front of the existing machine sequence $b[]$ and update the availability of machine type. The linear machine type sequence $(b[])$ after second product is 4-5-2-3-9-8-1-2.

Step III: Next $\left(5^{\text {th }}\right)$ product machine type sequence is 4-3-2-7-6. Machine type numbers (mno) 4 and 3 are unassigned and hence, add the machine types in front of $b[]$ and update the availability of machine type. Machine type number (mno) 2 is assigned and available in the existing sequence. Next machine types 7 and 6 are unassigned and unavailable in the existing machine sequence $b[]$ and hence, add the back side of the existing machine sequence $b[]$ and update the availability of machine type . The linear machine type sequence $(b[])$ after $5^{\text {th }}$ product is 4-3-4-5-2-3-9-8-1-2-7-6.

Step IV: Next $\left(3^{\text {rd }}\right)$ product machine type sequence is 1-7-6-2-4. Machine type numbers (mno) 1,7 and 6 are unassigned and hence, add the machine types in front of $b[]$ and update the availability of machine type. Machine type number (mno) 2 is assigned and available in the existing sequence. Next machine type 4 is unassigned and unavailable in the existing machine sequence $b[]$ and hence, machine type 4 is add the back side of the existing machine sequence $b[]$ and update the availability of machine type . The linear machine type sequence $(b[])$ after $3^{\text {rd }}$ product is 1-7-6-4-3-4-5-2-3-9-8-1-2-7-6-4.

Step V: Next $\left(6^{\text {th }}\right)$ product machine type sequence is 6-7-8-5. Machine type number (mno) 6 is unassigned and hence, add the machine type in front of $b[]$ and update the availability of machine type. Machine type numbers (mno) 7 and 8 are assigned and available in the existing sequence. Next machine type 5 is unassigned and unavailable in the existing machine sequence $b[]$ and hence, machine type 5 is add the back side of the existing machine sequence $b[]$ and update the availability of machine type . The linear machine type sequence $(b[])$ after $6^{\text {th }}$ product is 6-1-7-6-4-3-4-5-2-3-9-8-1-2-7-6-4-5.

Step VI: Next $\left(4^{\text {th }}\right)$ product machine type sequence is 3-6-5-1. Machine type number (mno) 3 is unassigned and hence, add the machine type in front of $b[]$ and update the availability of machine type. Machine type numbers (mno) 6,5 and 1 are assigned and available in the existing sequence. Hence, the linear machine type sequence $(b[])$ after $4^{\text {th }}$ product is 3-6-1-7-6-4-3-4-5-2-3-9-8-1-2-7-6-4-5. 
Step VII: Last $\left(7^{\text {th }}\right)$ product machine type sequence is 1-2-3-4. Machine type number (mno) 1 is unassigned and hence, add the machine type in front of $b[]$ and update the availability of machine type. Machine type numbers (mno) 2,3 and 4 are assigned and available in the existing sequence. Hence, the linear machine type sequence $(b[])$ after the last product is 1-3-6-1-7-6-4-3-4-5-2-3-9-8-1-2-7-6-4-5.

The total number of machines (nms) in the linear machine sequence is 20 . The flow distance (fd) and the total investment cost of machines(tc) and total material handling cost (tmhc) for the above linear machine sequence are listed in Tables 5, 6 and 7. 
Table 5. Determination of flow distance for the final machine sequence of product sequence 1-2-5-3-6-4-7 (Initial solution).

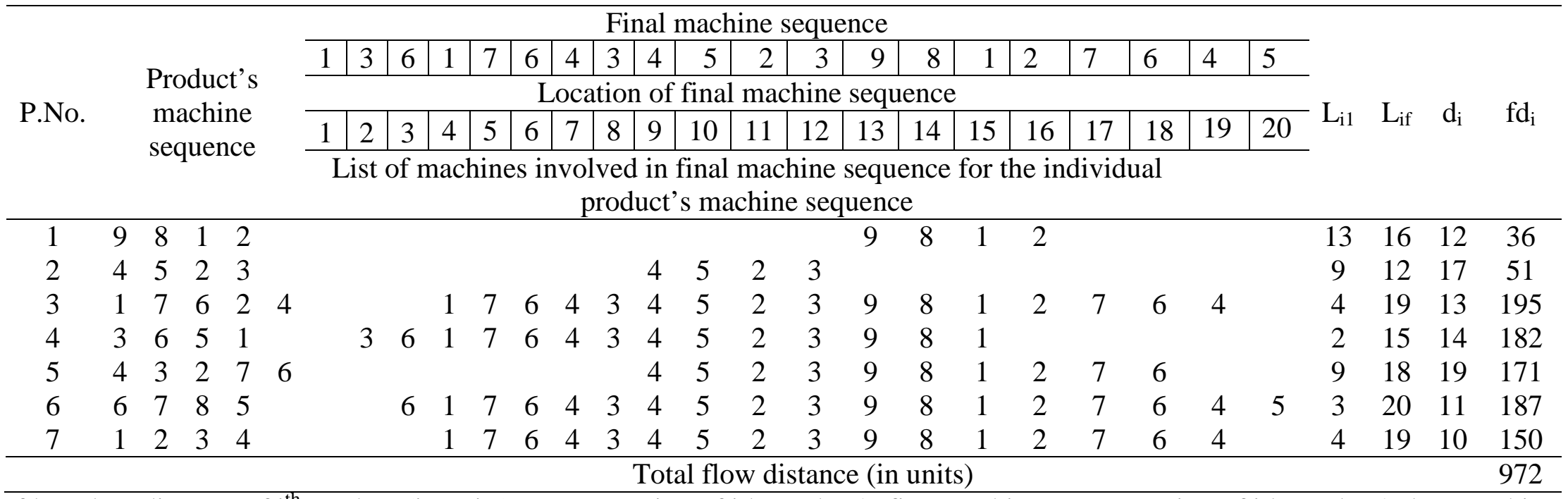

$\mathrm{fd}_{\mathrm{i}}-$ Flow distance of $\mathrm{i}^{\text {th }}$ product (in units); $\mathrm{L}_{\mathrm{i} 1}$ - Location of ith product's first machine; $\mathrm{L}_{\mathrm{if}}$ - Location of ith product's last machine

Table 6. Determination of total investment cost of machines for the final machine sequence of product sequence 1-2-5-3-6-4-7 (Initial solution).

\begin{tabular}{|c|c|c|c|c|c|c|c|c|c|c|}
\hline Machine type & 1 & 2 & 3 & 4 & 5 & 6 & 7 & 8 & 9 & Total \\
\hline $\begin{array}{l}\text { No. of machine type available in final } \\
\text { sequence }\end{array}$ & 3 & 2 & 3 & 3 & 2 & 3 & 2 & 1 & 1 & $\begin{array}{l}\text { investment } \\
\text { cost of }\end{array}$ \\
\hline Cost of machine type & 12554 & 32457 & 17685 & 19658 & 30254 & 22887 & 27889 & 19651 & 18357 & $\begin{array}{l}\text { machines } \\
\text { in Rs. }\end{array}$ \\
\hline Investment cost of each machine types & 37662 & 64914 & 53055 & 58974 & 60508 & 68661 & 55778 & 19651 & 18357 & $4,37,560$ \\
\hline
\end{tabular}


Table 7. Determination of total material handling cost for the final machine sequence of product sequence 1-2-5-3-6-4-7 (Initial solution).

\begin{tabular}{ccc}
\hline P.No. & Machine sequence & Material handling cost in Rs. \\
\hline 1 & $9-8-1-2$ & 159 \\
2 & $4-5-2-3$ & 130 \\
3 & $1-7-6-2-4$ & 737 \\
4 & $3-6-5-1$ & 641 \\
5 & $4-3-2-7-6$ & 430 \\
6 & $6-7-8-5$ & 823 \\
7 & $1-2-3-4$ & 737 \\
\hline \multicolumn{2}{c}{ Total material handling cost } \\
\hline
\end{tabular}

\subsection{Implementation of tabu search algorithm:}

In Tabu Search, the initial solution is generated as per the heuristic approach explained in section 5.1 for the random combination of product's sequence (1-2-5-3-6-4-7) and it is illustrated in Table 8. The normalized values of total flow distance, total number of machines, total investment cost of machines, total material handling cost, and average fitness factor are calculated based on the equations 10 to 15 respectively.

In the first stage, the neighbors are generated with a size of $(n p-2)$ based on interchanging the position of products within the initial sequence. After (np-2) iterations, select the minimum average fitness factor value among the solution and the corresponding product's sequence is moved to the tabu list.

In the second stage, the product's sequence in the tabu list is taken as an initial solution and formulate the neighbors similar to the first stage. Here, again (np-2) iterations are executed and the product's sequence for the corresponding minimum average fitness factor value has moved to the tabu list. The iteration is repeated upto the stopping criteria either there is no further change in fittnes value of consecuite iterations or a given number of iterations. The evaluation details of the first two stages are listed in Table 9. After that, the final solution and the corresponding product's sequence may get from the tabu list which has minimum average fitness factor value. The $\mathrm{C}$ program developed for this purpose will stop automatically after getting the final solution from the tabu list. 
Table 8. Initial solution in tabu search.

\begin{tabular}{|c|c|c|c|c|c|c|c|c|c|}
\hline $\begin{array}{l}\text { Product } \\
\text { Sequence }\end{array}$ & $\begin{array}{l}\text { tc in } \\
\text { Rs. }\end{array}$ & $\begin{array}{l}\text { tmhe } \\
\text { In Rs. }\end{array}$ & $\mathrm{nms}$ & $\mathrm{fd}$ & $\begin{array}{c}\text { Normalized } \\
\text { value of tc } \\
\text { N1 }\end{array}$ & $\begin{array}{c}\text { Normalized } \\
\text { value of tmhc } \\
\text { N2 }\end{array}$ & $\begin{array}{c}\text { Normalized } \\
\text { value of nms } \\
\text { N3 }\end{array}$ & $\begin{array}{c}\text { Normalized } \\
\text { value of fd } \\
\text { N4 }\end{array}$ & $\begin{array}{l}\text { Avg. fitness } \\
\text { factor }\end{array}$ \\
\hline $1-2-5-3-6-4-7$ & 437560 & 3657 & 20 & 972 & 0.763206 & 0.968669 & 0.75 & 0.793162 & 0.81876 \\
\hline
\end{tabular}

Table 9. Evaluation details of the first two stages in tabu search.

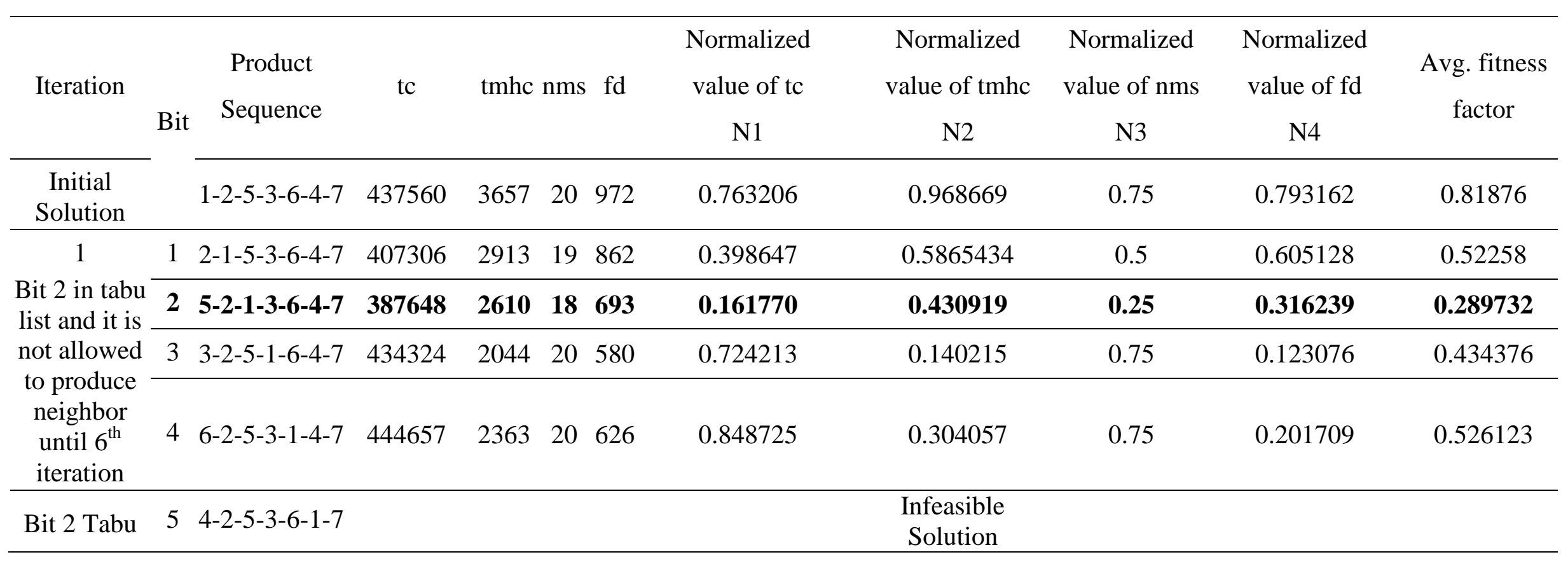




\begin{tabular}{|c|c|c|c|c|c|c|c|c|c|c|c|}
\hline \multirow{4}{*}{$\begin{array}{c}2 \\
\text { Bit } 1 \text { in tabu } \\
\text { list and it is } \\
\text { not allowed } \\
\text { to produce } \\
\text { neighbor } \\
\text { until } 7^{\text {th }} \\
\text { iteration }\end{array}$} & 6 & $7-2-1-3-6-4-5$ & & & & & & $\begin{array}{c}\text { Infeasible } \\
\text { Solution }\end{array}$ & & & \\
\hline & 1 & $2-5-1-3-6-4-7$ & 407306 & 2742 & 19 & 695 & 0.398647 & 0.498715 & 0.5 & 0.31965 & 0.429256 \\
\hline & 3 & $3-2-1-5-6-4-7$ & 434324 & 2084 & 20 & 621 & 0.724213 & 0.160760 & 0.75 & 0.193162 & 0.457034 \\
\hline & 4 & $6-2-1-3-5-4-7$ & 457211 & 2424 & 21 & 738 & 1 & 0.335387 & 1 & 0.393162 & 0.682138 \\
\hline Bit 1 tabu & 5 & $4-2-1-3-6-5-7$ & & & & & & $\begin{array}{l}\text { Infeasible } \\
\text { Solution }\end{array}$ & & & \\
\hline
\end{tabular}

The optimal solution and its corresponding product sequence evaluated by the tabu search algorithm is listed in Table 10 . The optimal linear machine sequence is 3-6-5-1-7-6-9-8-1-4-5-2-3-4-3-2-7-6 for the product sequence of 5-2-1-3-4-6-7.

Table 10. Optimal solution and its product sequence.

\begin{tabular}{ccccc}
\hline $\begin{array}{c}\text { Product } \\
\text { Sequence }\end{array}$ & tc & tmhc & nms & fd \\
\hline $5-2-1-3-4-6-7$ & 405348 & 1998 & 18 & 508 \\
\hline
\end{tabular}




\section{Computation results and discussions:}

A good number of randomly generated problems and as well as problems discussed in Pillai et al. (2005), Chen et al. (2001), and Siva Kumar et al. (2011) have also been solved using the proposed methodology. Input data, such as the number of products and their machine type sequences and product demand, are listed in Appendix Table A1. The number of machine types and their duplicate numbers are listed in Appendix Table A2. The cost of individual machine types is listed in Appendix Table A3. The material handling cost between machine to machine for problems 1-7 are listed in Appendix Tables A4-A10 repectively. The final linear machine sequence, product sequence, total flow distance, total investment cost of machines, total material handling cost and total number of machines in the final linear sequence are presented in Table 11.

As compared with the problems and solutions discussed in Pillai et al, Chen et al and Siva Kumar et al, the proposed algorithm yields the best linear sequence of machines which minimizes the total flow distance in units, total number of machines, total investment cost of machines and total material handling cost due to the following reasons.

a. Machines are assigned not on the basis of the descending order of the flow distance of a product's sequence.

b. The number of machines used in every machine type in the final linear machine sequence is reduced.

c. The unassigned machine types are incorporated at the front or back flow of the existing machine sequence, depending on availability.

d. If one of the machine types is assigned and it is available in the existing sequence, its availability in this sequence is verified even if the remaining machine types are unassigned. If any of the remaining machine types are unavailable in the existing sequence and are unassigned, then the machine type is incorporated at the back flow of the existing sequence without affecting the previous product machine type sequences. 
Table 11. Computation results.

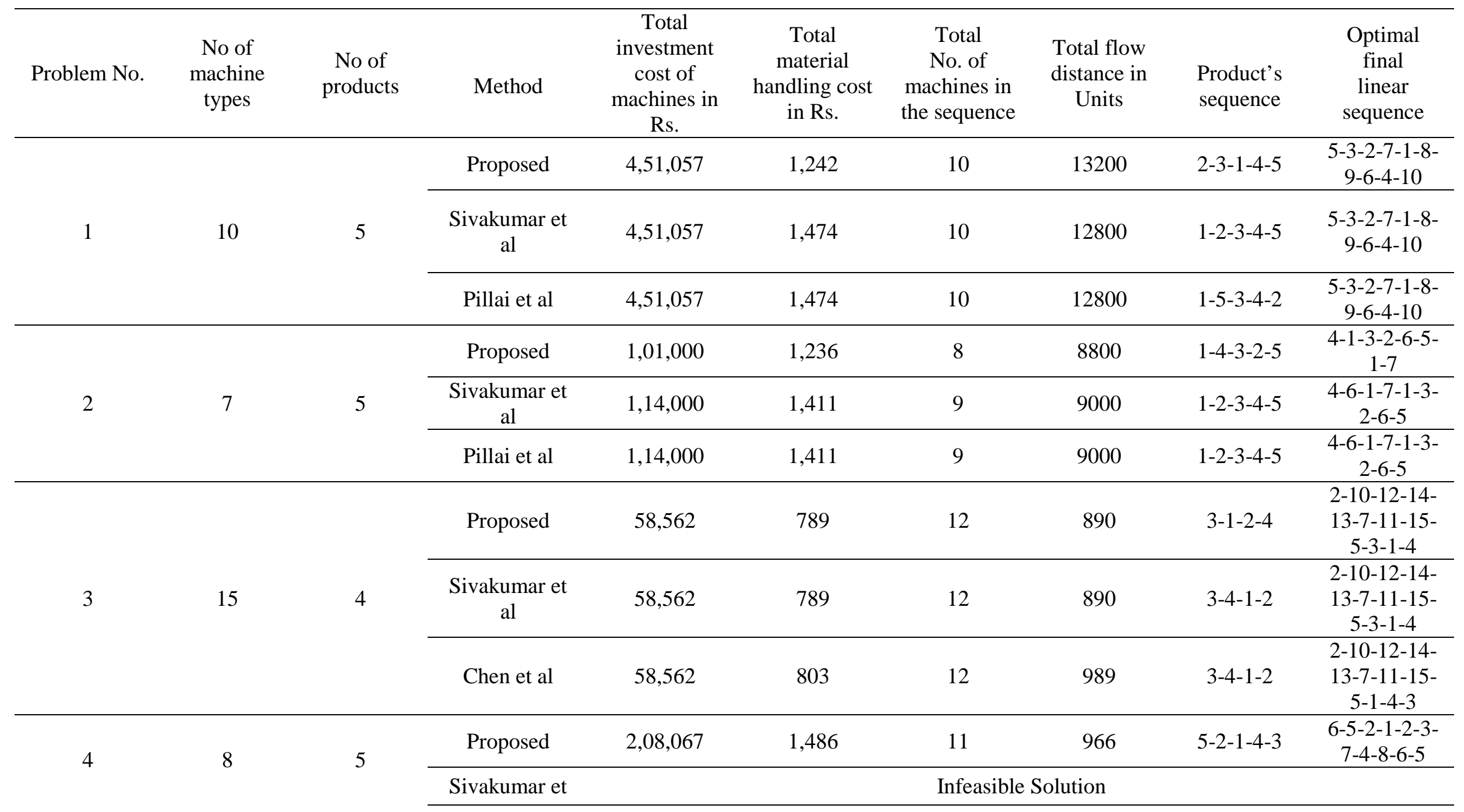


al

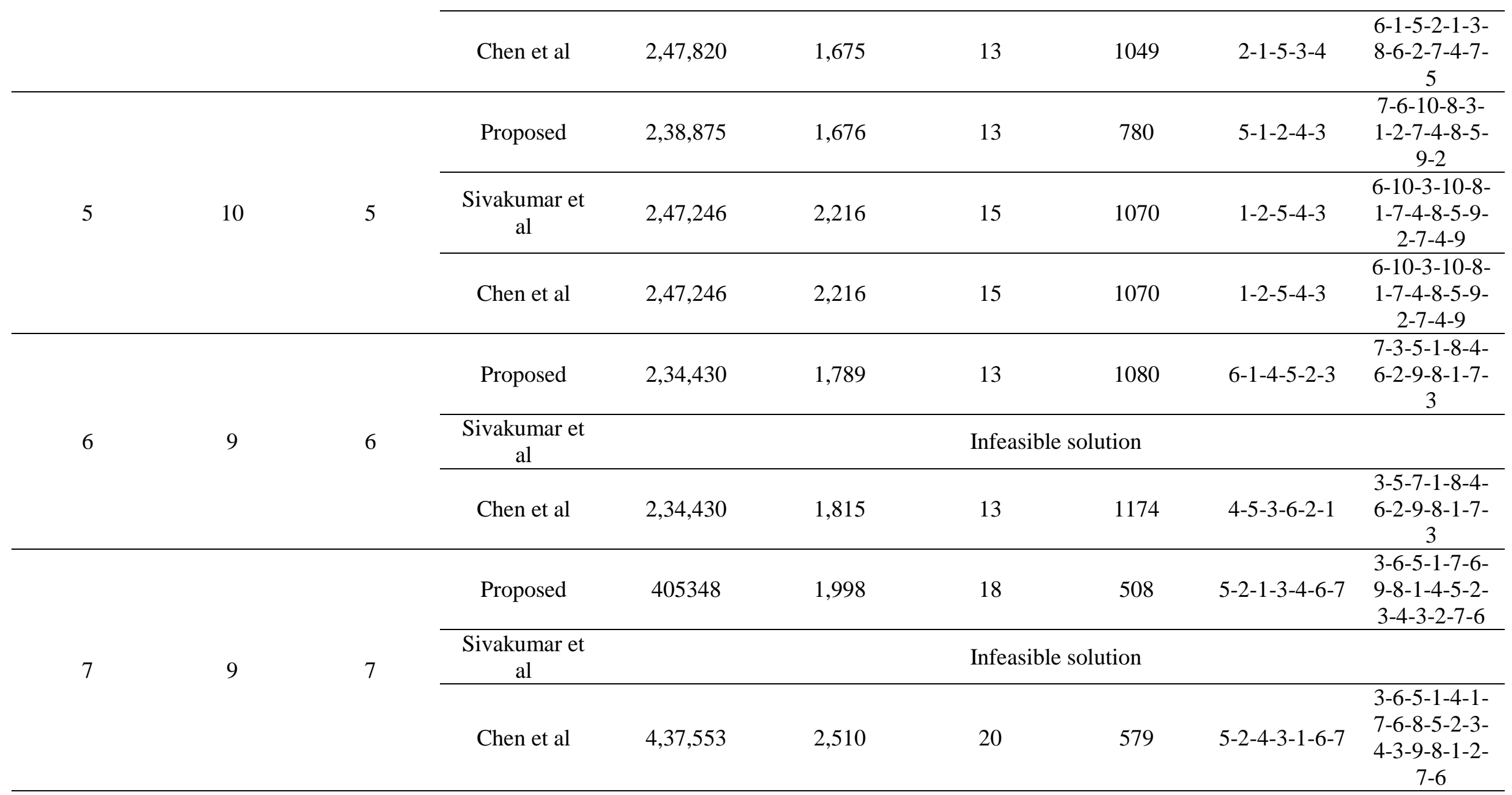




\section{Conclusion and future work:}

The linear sequence of machines in a layout design determines the flow distance and investment cost of machines for multi-products of different operation sequences with a single or limited number of duplicate machines type. This work proposed a tabu search algorithm to construct a linear sequence of machines that minimizes the total flow distance in units, total investment cost of machines in final linear sequence of machines, total material handling cost and total number of machine types arranged in the final linear sequence. It is concluded from Table 11 that the proposed method is highly efficient compared with other approaches. Other than the problems discussed in the literature, a good number of problems were generated and experimented with the proposed algorithm, and the obtained results were favorable when compared with previous approaches. As the extension of the present work, it is possible to solve by Particle Swarm Optimization tool and compared with the results of the present work.

\section{Appendices}

Table A1. Operation sequences and product demand of example problems

\begin{tabular}{|c|c|c|c|}
\hline Problem no & Products & Operation sequence & Product demand \\
\hline \multicolumn{4}{|c|}{ Literature Problems } \\
\hline \multirow{5}{*}{$\begin{array}{c}1 \\
\text { Pillai et al }\end{array}$} & 1 & $1-8-9-6-4$ & 700 \\
\hline & 2 & $5-3-2-7$ & 600 \\
\hline & 3 & $5-3-2-9$ & 500 \\
\hline & 4 & $3-7-6-4$ & 400 \\
\hline & 5 & $3-2-7-9-10$ & 300 \\
\hline \multirow{5}{*}{$\begin{array}{c}2 \\
\text { Pillai et al }\end{array}$} & 1 & $1-3-2-6-5$ & 800 \\
\hline & 2 & $4-6-1-7$ & 400 \\
\hline & 3 & $4-1-6-5$ & 300 \\
\hline & 4 & $4-3-2-5$ & 200 \\
\hline & 5 & $4-1-3-2$ & 100 \\
\hline \multirow{4}{*}{$\begin{array}{c}3 \\
\text { Chen et al }\end{array}$} & 1 & 14-13-7-15 & 34 \\
\hline & 2 & $2-10-12-13$ & 29 \\
\hline & 3 & $11-15-5-3$ & 94 \\
\hline & 4 & $15-5-1-4$ & 89 \\
\hline \multicolumn{4}{|c|}{ Generated Problems } \\
\hline \multirow[t]{5}{*}{ 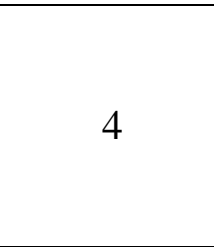 } & 1 & $6-2-7-4$ & 40 \\
\hline & 2 & $5-2-1-3$ & 46 \\
\hline & 3 & $6-2-3-5$ & 30 \\
\hline & 4 & $3-8-6-5$ & 17 \\
\hline & 5 & $1-2-3-7$ & 39 \\
\hline \multirow{5}{*}{5} & 1 & $1-7-4-8-5$ & 36 \\
\hline & 2 & $10-8-1-9-2$ & 35 \\
\hline & 3 & $7-8-4-9$ & 1 \\
\hline & 4 & $6-10-3-2$ & 28 \\
\hline & 5 & $3-1-2-7$ & 33 \\
\hline \multirow{3}{*}{6} & 1 & $4-6-8-1$ & 8 \\
\hline & 2 & $7-1-8-2$ & 15 \\
\hline & 3 & $5-6-9-8-3$ & 32 \\
\hline
\end{tabular}




\begin{tabular}{llll}
4 & $3-5-1-8$ & 50 \\
5 & $5-9-8-1-7$ & 42 \\
6 & $4-6-2-9$ & 29 \\
\hline 1 & $9-8-1-2$ & 12 \\
2 & $4-5-2-3$ & 17 \\
7 & $1-7-6-2-4$ & 13 \\
& 3 & $3-6-5-1$ & 14 \\
& 4 & $4-3-2-7-6$ & 19 \\
& 5 & $6-7-8-5$ & 11 \\
6 & $1-2-3-4$ & 10 \\
\hline
\end{tabular}

Table A2. Machine types and its duplicates for the example problems

\begin{tabular}{|c|c|c|c|c|c|c|c|c|c|c|c|c|c|c|c|}
\hline \multirow{2}{*}{$\begin{array}{c}\text { Problem } \\
\text { No. }\end{array}$} & \multicolumn{15}{|c|}{ Machines Types } \\
\hline & 1 & 2 & 3 & 4 & 5 & 6 & 7 & 8 & 9 & 10 & 11 & 12 & 13 & 14 & 15 \\
\hline 1 & 1 & 1 & 1 & 1 & 1 & 1 & 1 & 1 & 1 & 1 & & & & & \\
\hline 2 & 2 & 1 & 1 & 1 & 1 & 2 & 1 & & & & & & & & \\
\hline 3 & 1 & 1 & 1 & 2 & 1 & 2 & 2 & 2 & 2 & 2 & 1 & 2 & 1 & 1 & 1 \\
\hline 4 & 2 & 2 & 1 & 1 & 2 & 2 & 2 & 1 & & & & & & & \\
\hline 5 & 1 & 2 & 1 & 2 & 1 & 1 & 2 & 2 & 2 & 2 & & & & & \\
\hline 6 & 2 & 1 & 2 & 2 & 1 & 1 & 2 & 2 & 2 & & & & & & \\
\hline 7 & 3 & 2 & 3 & 3 & 2 & 3 & 2 & 3 & 3 & & & & & & \\
\hline
\end{tabular}

Table A3. Machine types and its cost for the example literature problems

\begin{tabular}{crrrrrrr}
\hline Machines & \multicolumn{7}{c}{ Problem No. } \\
\cline { 2 - 8 } Types & 1 & \multicolumn{1}{c}{2} & \multicolumn{1}{c}{ 3 } & \multicolumn{1}{c}{5} & \multicolumn{1}{c}{6} & \multicolumn{1}{c}{7} \\
\hline 1 & 84,565 & 10,000 & 8,788 & 16,273 & 19,323 & 24,121 & 12,554 \\
2 & 74,325 & 15,000 & 6,589 & 27,409 & 21,824 & 4,546 & 32,457 \\
3 & 59,874 & 16,000 & 3,512 & 15,276 & 27,077 & 25,742 & 17,685 \\
4 & 39,998 & 12,000 & 6,541 & 3,135 & 3,809 & 27,159 & 19,658 \\
5 & 47,775 & 11,000 & 3,254 & 19,058 & 19,080 & 26,738 & 30,254 \\
6 & 22,225 & 13,000 & 9,874 & 21,828 & 13,912 & 18,822 & 22,887 \\
7 & 14,411 & 14,000 & 6,547 & 23,400 & 28,026 & 21,612 & 27,889 \\
8 & 15,455 & & 8,541 & 13,393 & 14,794 & 979 & 19,651 \\
9 & 34,545 & & 3,256 & & 13,931 & 12,257 & 18,357 \\
10 & 57,884 & & 1,111 & & 12,455 & & \\
11 & & 2,222 & & & & \\
12 & & 3,333 & & & & \\
13 & & 4,445 & & & & \\
14 & & 5,554 & & & & \\
15 & & 6,666 & & & & \\
\hline
\end{tabular}

Table A4 - A10. Material handling cost between machine to machine for the problems 1-7

\begin{tabular}{ccccccccccc}
\hline P.No. 1 & 1 & 2 & 3 & 4 & 5 & 6 & 7 & 8 & 9 & 10 \\
\hline 1 & 0 & 33 & 59 & 41 & 37 & 43 & 68 & 67 & 26 & 65 \\
\hline
\end{tabular}




\begin{tabular}{ccccccccccc}
\hline 2 & 54 & 0 & 61 & 67 & 53 & 55 & 47 & 32 & 68 & 59 \\
3 & 61 & 63 & 0 & 32 & 35 & 57 & 33 & 32 & 45 & 63 \\
4 & 37 & 43 & 52 & 0 & 58 & 58 & 31 & 69 & 55 & 38 \\
5 & 52 & 55 & 32 & 41 & 0 & 65 & 54 & 53 & 50 & 45 \\
6 & 31 & 66 & 55 & 34 & 46 & 0 & 37 & 38 & 49 & 55 \\
7 & 60 & 54 & 51 & 49 & 32 & 65 & 0 & 48 & 57 & 61 \\
8 & 65 & 48 & 63 & 54 & 35 & 54 & 31 & 0 & 64 & 36 \\
9 & 58 & 59 & 55 & 45 & 32 & 42 & 53 & 44 & 0 & 33 \\
10 & 32 & 45 & 47 & 58 & 62 & 60 & 58 & 45 & 62 & 0 \\
\hline & & & & & & & & & & \\
\hline P.No. 2 & 1 & 2 & 3 & 4 & 5 & 6 & 7 \\
\hline 1 & & 0 & 64 & 54 & 32 & 64 & 55 & 47 \\
2 & & 54 & 0 & 45 & 47 & 53 & 57 & 36 \\
3 & & 38 & 47 & 0 & 43 & 57 & 52 & 31 \\
4 & 42 & 48 & 63 & 0 & 39 & 47 & 34 \\
5 & & 44 & 36 & 55 & 37 & 0 & 36 & 49 \\
6 & 40 & 36 & 49 & 67 & 61 & 0 & 42 \\
7 & & 39 & 43 & 52 & 57 & 54 & 61 & 0 \\
\hline
\end{tabular}

\begin{tabular}{|c|c|c|c|c|c|c|c|c|c|c|c|c|c|c|c|}
\hline $\begin{array}{c}\text { P.No. } \\
3\end{array}$ & 1 & 2 & 3 & 4 & 5 & 6 & 7 & 8 & 9 & 10 & 11 & 12 & 13 & 14 & 15 \\
\hline 1 & 0 & 54 & 61 & 37 & 52 & 31 & 60 & 65 & 58 & 32 & 47 & 63 & 69 & 54 & 35 \\
\hline 2 & 33 & 0 & 63 & 43 & 55 & 66 & 54 & 48 & 59 & 45 & 58 & 61 & 55 & 53 & 44 \\
\hline 3 & 59 & 61 & 0 & 52 & 32 & 55 & 51 & 63 & 55 & 47 & 69 & 57 & 38 & 50 & 53 \\
\hline 4 & 41 & 67 & 32 & 0 & 41 & 34 & 49 & 54 & 45 & 58 & 49 & 53 & 68 & 45 & 47 \\
\hline 5 & 37 & 53 & 35 & 58 & 0 & 46 & 32 & 35 & 32 & 62 & 52 & 56 & 62 & 35 & 65 \\
\hline 6 & 43 & 55 & 57 & 58 & 65 & 0 & 65 & 54 & 42 & 60 & 37 & 46 & 68 & 47 & 57 \\
\hline 7 & 68 & 47 & 33 & 31 & 54 & 37 & 0 & 31 & 53 & 58 & 33 & 58 & 40 & 64 & 49 \\
\hline 8 & 67 & 32 & 32 & 69 & 53 & 38 & 48 & 0 & 44 & 45 & 53 & 35 & 69 & 62 & 52 \\
\hline 9 & 26 & 68 & 45 & 55 & 50 & 49 & 57 & 64 & 0 & 62 & 65 & 63 & 52 & 53 & 47 \\
\hline 10 & 65 & 59 & 63 & 38 & 45 & 55 & 61 & 36 & 33 & 0 & 47 & 55 & 49 & 38 & 59 \\
\hline 11 & 68 & 32 & 53 & 68 & 35 & 67 & 33 & 41 & 32 & 32 & 0 & 50 & 44 & 58 & 54 \\
\hline 12 & 66 & 51 & 61 & 62 & 47 & 48 & 36 & 52 & 41 & 61 & 50 & 0 & 36 & 56 & 33 \\
\hline 13 & 31 & 50 & 55 & 68 & 64 & 49 & 68 & 65 & 37 & 60 & 45 & 69 & 0 & 31 & 32 \\
\hline 14 & 33 & 40 & 68 & 40 & 36 & 50 & 69 & 67 & 47 & 49 & 52 & 59 & 67 & 0 & 47 \\
\hline 15 & 41 & 52 & 47 & 65 & 62 & 36 & 48 & 49 & 54 & 51 & 64 & 47 & 57 & 62 & 0 \\
\hline \multicolumn{2}{|c|}{ P.No. 4} & \multicolumn{2}{|c|}{1} & \multicolumn{2}{|c|}{2} & 3 & & 4 & \multicolumn{2}{|c|}{5} & \multicolumn{2}{|l|}{6} & 7 & \multicolumn{2}{|c|}{8} \\
\hline \multicolumn{2}{|c|}{1} & \multicolumn{2}{|c|}{0} & \multicolumn{2}{|c|}{58} & 58 & & 31 & \multicolumn{2}{|c|}{69} & \multicolumn{2}{|l|}{55} & 38 & \multicolumn{2}{|c|}{68} \\
\hline \multicolumn{2}{|c|}{2} & \multicolumn{2}{|c|}{41} & \multicolumn{2}{|c|}{0} & 65 & & 54 & \multicolumn{2}{|c|}{53} & \multicolumn{2}{|l|}{50} & 45 & \multicolumn{2}{|c|}{35} \\
\hline \multicolumn{2}{|c|}{3} & \multicolumn{2}{|c|}{34} & \multicolumn{2}{|c|}{46} & 0 & & 37 & \multicolumn{2}{|c|}{38} & \multicolumn{2}{|l|}{49} & 55 & \multicolumn{2}{|c|}{67} \\
\hline \multicolumn{2}{|c|}{4} & & 49 & 32 & & 65 & & 0 & \multicolumn{2}{|c|}{48} & 57 & & 61 & \multicolumn{2}{|c|}{33} \\
\hline \multicolumn{2}{|c|}{5} & & 54 & 35 & & 54 & & 31 & \multicolumn{2}{|c|}{0} & 64 & & 36 & & 11 \\
\hline 6 & & & 45 & 32 & & 42 & & 53 & 4 & & 0 & & 33 & & 32 \\
\hline 7 & & & 58 & 62 & & 60 & & 58 & 4 & & 62 & & 0 & & 32 \\
\hline 8 & & & 49 & 52 & & 37 & & 33 & 5 & & 65 & & 47 & & 0 \\
\hline
\end{tabular}




\begin{tabular}{ccccccccccc}
\hline P.No. 5 & 1 & 2 & 3 & 4 & 5 & 6 & 7 & 8 & 9 & 10 \\
\hline 1 & 0 & 52 & 32 & 55 & 51 & 63 & 55 & 47 & 69 & 57 \\
2 & 32 & 0 & 41 & 34 & 49 & 54 & 45 & 58 & 49 & 53 \\
3 & 35 & 58 & 0 & 46 & 32 & 35 & 32 & 62 & 52 & 56 \\
4 & 57 & 58 & 65 & 0 & 65 & 54 & 42 & 60 & 37 & 46 \\
5 & 33 & 31 & 54 & 37 & 0 & 31 & 53 & 58 & 33 & 58 \\
6 & 32 & 69 & 53 & 38 & 48 & 0 & 44 & 45 & 53 & 35 \\
7 & 45 & 55 & 50 & 49 & 57 & 64 & 0 & 62 & 65 & 63 \\
8 & 63 & 38 & 45 & 55 & 61 & 36 & 33 & 0 & 47 & 55 \\
9 & 53 & 68 & 35 & 67 & 33 & 41 & 32 & 32 & 0 & 50 \\
10 & 61 & 62 & 47 & 48 & 36 & 52 & 41 & 61 & 50 & 0 \\
\hline
\end{tabular}

\begin{tabular}{cccccccccc}
\hline P.No. 6 & 1 & 2 & 3 & 4 & 5 & 6 & 7 & 8 & 9 \\
\hline 1 & 0 & 58 & 58 & 31 & 69 & 55 & 38 & 68 & 62 \\
2 & 41 & 0 & 65 & 54 & 53 & 50 & 45 & 35 & 47 \\
3 & 34 & 46 & 0 & 37 & 38 & 49 & 55 & 67 & 48 \\
4 & 49 & 32 & 65 & 0 & 48 & 57 & 61 & 33 & 36 \\
5 & 54 & 35 & 54 & 31 & 0 & 64 & 36 & 41 & 52 \\
6 & 45 & 32 & 42 & 53 & 44 & 0 & 33 & 32 & 41 \\
7 & 58 & 62 & 60 & 58 & 45 & 62 & 0 & 32 & 61 \\
8 & 49 & 52 & 37 & 33 & 53 & 65 & 47 & 0 & 50 \\
9 & 53 & 56 & 46 & 58 & 35 & 63 & 55 & 50 & 0 \\
\hline
\end{tabular}

\begin{tabular}{cccccccccc}
\hline P.No. 7 & 1 & 2 & 3 & 4 & 5 & 6 & 7 & 8 & 9 \\
\hline 1 & 0 & 41 & 34 & 49 & 54 & 45 & 58 & 49 & 53 \\
2 & 58 & 0 & 46 & 32 & 35 & 32 & 62 & 52 & 56 \\
3 & 58 & 52 & 0 & 65 & 54 & 42 & 60 & 37 & 46 \\
4 & 31 & 32 & 37 & 0 & 31 & 53 & 58 & 33 & 58 \\
5 & 69 & 55 & 38 & 48 & 0 & 44 & 45 & 53 & 35 \\
6 & 55 & 51 & 49 & 57 & 64 & 0 & 62 & 65 & 63 \\
7 & 38 & 63 & 55 & 61 & 36 & 33 & 0 & 47 & 55 \\
8 & 68 & 55 & 67 & 33 & 41 & 32 & 32 & 0 & 50 \\
9 & 33 & 53 & 65 & 47 & 52 & 41 & 61 & 50 & 0 \\
\hline
\end{tabular}

\section{References:}

1. André, R.S. and Amaral., 2008. An exact approach to the one-dimensional facility layout problem. Operations Research, 56, 1026-1033.

2. Aneke, N.A. and Carrie, A.S., 1986. A design technique for layout of multi-product flowlines. International Journal of Production Research, 24, 471- 481.

3. Bhaba R. Sarker, Ahmad Diponegoro., 2003. Flow distance reduction for a multiproduct flowline with sets of identical machines, European Journal of Operations Research, 147, 591-612. 
4. Booncharoen Sirinaovakul., 1996. An analysis of computer-aided facility layout techniques. International Journal of Computer Integrated Manufacturing, 9(4), 260-264.

5. Braglia, M., 1997. Heuristics for single-row layout problems in flexible manufacturing problems. Production Planning and Control, 8, 558-567.

6. Chen, D.S., Wang, Q. and Chen, H.C., 2001. Linear sequencing for machine layouts by a modified simulated annealing. International Journal of Production Research, 39(8), 1721 - 1732.

7. Chrysostomos, F. and Vlachos, A., 2005. Optimal solution of linear machine layout problem using ant colony system. Proceedings of the 9th WSEAS International Conference on Computers, Athens, Greece: World Scientific and Engineering Academy and Society (WSEAS), 97.

8. Glover, F. and Laguna, M., 1997. Tabu Search. Boston/ Dordrecht/London: Kluwer Academic Publishers.

9. Hamed Samarghandi. and Kourosh Eshghi., 2010. An efficient tabu algorithm for the single row facility layout problem. European Journal of Operational Research, 205, 98-105.

10. Heragu, S.S. and Kusiak, A., 1990. Machine layout: An optimization and knowledge-based approach. International Journal of Production Research, 28, 615635.

11. Hicks, C., 2004. A genetic algorithm tool for designing manufacturing facilities in the capital goods industry. International Journal of Production Economics, 90(2), 199-211.

12. Ho, Y.C. and Moodie, C.L., 1998. Machine layout with a linear single-row flow path in an automated manufacturing system. Journal of Manufacturing Systems, 17(1), 1-22.

13. Houshyar, A. and McGinnis, L.F., 1990. A heuristic for assigning facilities to locations to minimize WIP travel distance in a linear facility. International Journal of Production Research, 28, 1485 - 1498.

14. Housni Djellab. and Michel Gourgand., 2001. A new heuristic procedure for the single-row facility layout problem. International Journal of Computer Integrated Manufacturing, 14 (3), 270-280.

15. Kouvelis, P. and Chiang, W.C., 1992. A simulated annealing procedure for single row layout problems in flexible manufacturing systems. International Journal of Production Research, 30, 717-732. 
16. Kouvelis, P. and Kim, M.W., 1992. Unidirectional loop network layout problem in automated manufacturing systems. Operation Research, 40, 533-550.

17. Lee, S.D., Huang, K.H. and Chiang, C.P., 2001. Configuring layout in unidirectional loop manufacturing systems. International Journal of Production Research, 39(6), 1183-1201.

18. Lin, M.T., 2009. The single-row machine layout problem in apparel manufacturing by hierarchical order-based genetic algorithm. International Journal of Clothing Science and Technology, 21(1), 31-43.

19. Lou Y. Liang. and Wen C. Chao., 2008. The strategies of tabu search technique for facility layout optimization. Automation in construction, 17, 657-669.

20. Nicholas, J., 2001. Competitive Manufacturing Management: Continuous Improvement, Lean Production and Customer Focused Quality. New Delhi: Tata McGraw Hill.

21. Pillai, V.M. and Gudivada, B.S., 2005. A simulated annealing algorithm for linear sequencing of machines for layout design. Sixth Int. Conference on Operations and Quantitative Management, August 9-11, 2005, Indore, India.

22. Ramazan Şahin and Orhan Türkbey., 2009. A simulated annealing algorithm to find approximate pareto optimal solutions for the multi-objective facility layout problem. In. J. Adv. Manuf. Techn., 41 (9-10), 1003-1018.

23. Satheesh Kumar, M., Asokan, P. and Kumanan, S., 2010. An artificial immune system-based algorithm to solve linear and loop layout problems in flexible manufacturing systems. International Journal of Product Development, 10(1-3), 165-179.

24. Singh, S.P. and Sharma, R.R.K., 2006. A review of different approaches to the facility layout problems. In. J. Adv. Manuf. Techn., 30 (5-6), 425-433.

25. Sivakumar, K., Balamurugan, C. and Ramabalan, S, 2011. Simultaneous optimal selection of design and manufacturing tolerance with alternative manufacturing process selection. Computer-Aided Design, 43, 207-218.

26. Siva Kumar, M., Islam M N., Lenin, N., Vignesh Kumar, D. and Ravindran, R, 2011. A simple heuristic for linear sequencing of machines in layout design. International Journal of Production Research, 49 (22), 6749-6768.

27. Solimanpur, M., Prem Vrat, Ravi Shankar, 2005. An ant algorithm for the single row layout problem in flexible manufacturing systems. Computers \& Operations Research, 32(3), 583-598.

28. Suresh, G. and Sahu, S., 1993. Multiobjective facility layout using simulated annealing. International Journal of Production Economics, 32, 239-254. 
29. Teo, Y.T. and Ponnambalam, S.G., 2008. A hybrid ACO/PSO heuristic to solve single row layout problem. CASE 2008. IEEE International Conference on Automation Science and Engineering 23-26 Aug. 2008, Washington, DC, USA. 597 $-602$.

30. Wang, T.Y., Lin, H.C. and Wu, K.B., 1998. An improved simulated annealing for facility layout problems in cellular manufacturing systems. Computers \& Industrial Engineering, 34(2), 309-319.

31. Wen-Chyuan Chiang and Chi Chiang., 1998. Intelligent local search strategies for solving facility layout problems with the quadratic assignment problem formulation. European Journal of Operational Research, 106, 457-488.

32. Zhou, J. and Irani, S.A., 2003. A new flow diagramming scheme for mapping and analysis of multi product flows in a facility. Journal of Integrated Design and Process Science, 7, 25-58. 